\title{
THERMOELASTIC PHENOMENA IN THE TRANSVERSALLY GRADED LAMINATES
}

\author{
J. JĘDRYSIAK ${ }^{*}$ AND E. PAZERA ${ }^{\dagger}$ \\ *Department of Structural Mechanics \\ Łódź University of Technology \\ al. Politechniki 6, 90-924 Łódź, Poland \\ e-mail: jarek@p.lodz.pl \\ ${ }^{\dagger}$ Department of Structural Mechanics \\ Łódź University of Technology \\ al. Politechniki 6, 90-924 Łódź, Poland \\ email: ewelina.pazera@p.lodz.pl
}

Key words: Thermoelasticity, Transversally Graded Laminates, Functionally Graded Materials, Tolerance Modelling, Asymptotic Modelling.

\begin{abstract}
The object of considerations is a laminate, made of two components nonperiodically distributed as microlaminas along one direction. In this note, two models describing the thermoelasticity problems are proposed: the tolerance model, taking into account the effect of the microstructure size on the overall behaviour of these laminates, and the asymptotic model, neglecting this effect. To obtain the governing equations of the tolerance and the asymptotic model, the tolerance averaging technique is applied.
\end{abstract}

\section{INTRODUCTION}

In this paper we deal with the problem of thermoelasticity in a two-phase laminate. The cells are composed of two sublayers of different material constituents. The thickness of the cells (cf. Fig. 1) is denoted by $l$ and is constant (so-called the uniform distribution of the cells). It is assumed that macroscopic properties of this laminate change continuously along one direction (perpendicular to the laminas). Hence, this object can be treated as made of a functional graded material (FGM), cf. Suresh and Mortensen [1]. Such laminates can be called transversally graded laminates (TG-type laminates), cf. Jędrysiak [2]. A fragment of the laminate of this kind is shown in Fig. 1.

Although usually in such composites basic cell cannot be simply defined and thermomechanical phenomena can be considered only with reference to micromechanical models with idealised geometries. The functionally graded laminates are not homogeneous in a macroscale, nonetheless in the analysis of various issues concerning these laminates, assumptions of idealisation can be similar to those applied to analyse composites with a periodic structure and their overall behaviour can be described by adopted and modified methods, which are used for macroscopically homogeneous composites. Some of the averaged methods adapted to analyse and determine TG-type laminates are discussed in Suresh and Mortensen [9] and Reiter et al. [7]. Between these methods, there are techniques 
based on the asymptotic homogenizations, cf. Jikov et al. [8] and methods using models with microlocal parameters, cf. Matysiak [10]. There are the alternative methods for functionally graded materials, such as a Green's function approach, cf. Kim and Noda [11], the Laplace transformation, cf. Ootao and Tanigawa [12], higher-order plate theory is proposed by Aboudi et al. [13] and reformulated by Bansal and Pindera [14], stochastic thermal stresses in objects with functionally graded materials properties are analysed by Chiba [15], a numerical homogenization technique is used by Schmauder and Weber [16], the boundary element method by Goldberg and Hopkins [17], meshless computational method is proposed by Sladek J. et al. [18], the finite difference method by Sadowski et al. [19]. Unfortunately, the most of the know approaches, do not take into account the effect of microstructure size on the overall behaviour of these laminates.
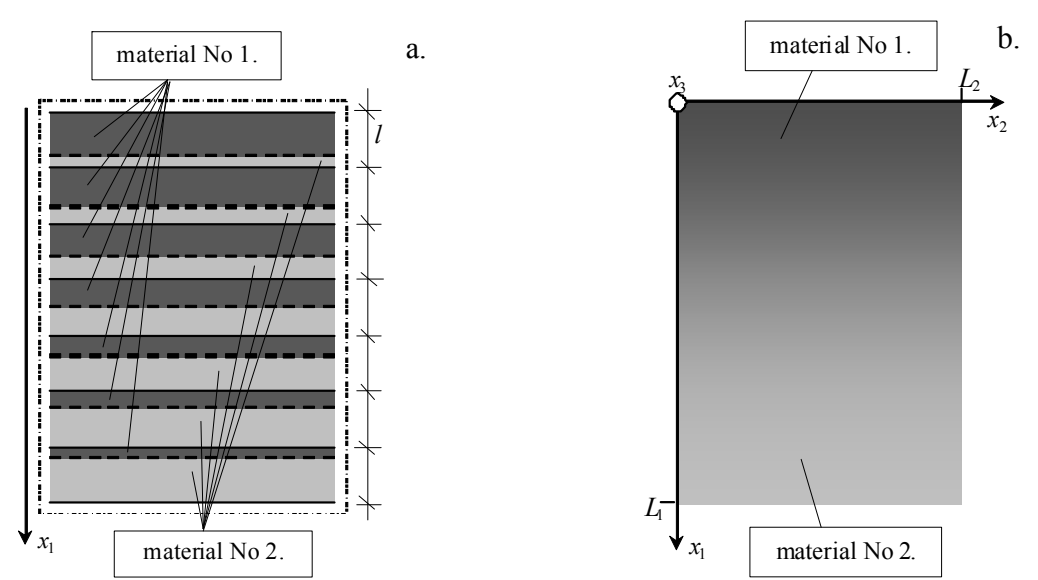

Figure 1: The cross-section of considered laminate: a. microstructure, b. macrostructure.

In order to obtain the averaged equations, taking into account this effect, the tolerance averaging technique is applied, proposed by Woźniak and Wierzbicki [15] to describe various thermo-mechanical phenomena in periodic composites. This way of modelling and the results of applying this technique for functionally graded media can be found in Jędrysiak [16], Woźniak [17], Woźniak et al. [18], Ostrowski and Michalak [19], Ostrowski [20]. TG-type laminates was also investigated, e.g. a heat conduction by Jędrysiak and Radzikowska [21]. The tolerance averaging technique replace equations with functional, highly-oscillating, tolerance-periodic and non-continuous coefficients by a system of differential equations with slowly-varying coefficients.

The main aim of this note is to obtain equations, describing thermoelasticity problems for TG-type laminates. The equations of two models are received. One of them is the tolerance model, which takes into account the effect of the microstructure size on the overall behaviour of the laminates with functionally graded material properties. And the second - the asymptotic model - which neglects this effect. Both of these models are based on concepts of the tolerance modelling. Applications of these models are shown in some examples. Applications of these models are shown in some examples. The problem of thermoelasticity in microperiodic structures was also considered by use of other mathematical models, approaches and shown in many papers. We shall mention here Refined Averaged Theory presented in paper of Baczyński [22] and Effective Modulus Theory in papers by Ignaczak [23]. 


\section{MODELLING FOUNDATIONS}

Let subscripts $i, j, . .$, run over $1,2,3$ (related to the coordinate system $O x_{1} x_{2} x_{3}$ and subscripts $\alpha, \beta, .$. , run over 2,3 (related to the coordinate system $O x_{2} x_{3}$ ). Denote: $\mathbf{x} \equiv\left(x_{1}, x_{2}\right), x \equiv x_{1}$, the time coordinate by $t$, derivatives respected to $x_{i}, x_{\alpha}, x$ by respectively $\partial_{i}, \partial_{\alpha}, \partial$ respectively, the laminate length along the $x$-axis by $H$, length dimensions along $x_{\alpha}$-axis by $L_{\alpha}$. Using denotations $\Omega \equiv(0, H), \Xi \equiv\left(0, L_{2}\right) \times\left(0, L_{3}\right)$, the laminate under consideration occupies in the physical space the region $\Omega \times \Xi$. This laminate is made of two materials distributed in $m$ laminas, the thickness of the lamina $(l)$ is constant $(H=m l)$ and can be called the microstructure parameter. It is assumed that condition $l<<$ is satisfied. Denote by $\Delta \equiv(-l / 2, l / 2)$ the basic cell in the interval $\Omega$. Every lamina $n$ is made of two homogeneous sublayers with thickness $l_{n}^{\prime}, l_{n}^{\prime \prime}$, depended on the argument $x$. The properties of sublayers are described by specific heats $c^{\prime}$ and $c^{\prime \prime}$, heat conduction tensors with components $k_{i j}^{\prime}, k_{i j}^{\prime \prime}$, mass densities $\rho^{\prime}, \rho^{\prime \prime}$, elasticity tensors with components $c_{i j k l}^{\prime}, c_{i j k l}^{\prime \prime}$, thermoelasticity tensors $b_{i j}^{\prime}, b_{i j}^{\prime \prime}$; where $i, j, k, l=1,2,3$. Denote material volume fractions in the $n^{\text {th }}$ lamina by $v_{n}^{\prime} \equiv l_{n}^{\prime} / l, v_{n}^{\prime \prime} \equiv l_{n}^{\prime \prime} / l$. We assume that the following conditions are satisfied: $\left|v_{n+1}^{\prime}-v_{n}^{\prime}\right|<<1$ and $v_{n}^{\prime}+v_{n}^{\prime \prime}=1$. It can be shown that sequences $\left\{v_{n}^{\prime}\right\},\left\{v_{n}^{\prime \prime}\right\}, n=1, \ldots, m$, can be approximated by continuous functions $v^{\prime}(\cdot), v^{\prime \prime}(\cdot)$. These functions can be called the fraction ratios of materials and describe gradation of material properties along the $x$-axis. The non-homogeneity ratio is denoted and defined by $v(\cdot) \equiv \sqrt{v^{\prime}(\cdot) v^{\prime \prime}(\cdot)}$. The unknown displacements along the $x_{i}$-axis are denoted by $u_{i}$ $(i=1,2,3)$ and the temperature field by $\Theta$.

The thermoelasticity problems for laminates with transversally graded material properties can be described by the know equations:

$$
\begin{aligned}
& \partial_{j}\left(c_{i j k l} \partial_{l} u_{k}\right)-\rho \ddot{u}_{i}=\partial_{j}\left(b_{i j} \Theta\right), \\
& \partial_{j}\left(k_{i j} \partial_{i} \Theta\right)-c \dot{\Theta}=T_{0} b_{i j} \partial_{j} \dot{u}_{i},
\end{aligned}
$$

where $T_{0}$ is a constant with a temperature dimension.

Unfortunately, all coefficients of the above equations (1), $k_{i j}, c, c_{i j k l}, b_{i j}$, $\rho$, are highlyoscillating, tolerance-periodic and non-continuous functions in $x$. By using the tolerance modelling we can replace these equations by differential equations with smooth, slowlyvarying functional coefficients, cf. Woźniak et al. [17].

\section{INTRODUCTORY CONCEPTS}

For this purpose, some basic concepts of the tolerance modelling, e.g. an averaging operator, a tolerance-periodic function, a slowly-varying function, a highly-oscillating function, are introduced below. The averaging operator for an arbitrary integrable function $f$, is defined by:

$$
<f>(x)=\frac{1}{l} \int_{x-l / 2}^{x+l / 2} f(y) d y, \quad x \in[l / 2, H-l / 2] .
$$

Denote $k^{\text {th }}$ gradient of function $f=f(x)$ by $\partial^{k} f, x \in \Omega, k=0,1, \partial^{0} f \equiv f$ and function defined in $\Omega \times R^{m}$ by $\widetilde{f}^{(k)}(\cdot$,$) . Function f \in H^{\alpha}(\Omega)$ is the tolerance-periodic function (with respect to cell 
$\Delta$ and tolerance parameter $\delta), f \in T P_{\delta}^{\alpha}(\Omega, \Delta)$, if for $k=0,1$, the following conditions hold:

a. $(\forall x \in \Omega)\left(\exists \tilde{f}^{(k)}(x ;) \in H^{0}(\Omega)\left[\left\|\left.\partial^{k} f\right|_{\Omega_{x}}(\cdot)-\widetilde{f}^{(k)}(x, ;)\right\|_{H^{0}\left(\Omega_{x}\right)} \leq \delta\right]\right.$,

b. $\int_{\Delta(\cdot)} \widetilde{f}^{(k)}(\cdot, y) d y \in C^{0}(\bar{\Omega})$,

where function $\widetilde{f}^{(k)}$ is the periodic approximation of $\partial^{k} f$ in $\Delta(x)=x+\Delta, x \in \Omega, k=0,1$.

Function $F \in H^{\alpha}(\Omega)$ is the slowly-varying function (with respect to cell $\Delta$ and tolerance parameter $\delta), F \in S V_{\delta}^{\alpha}(\Omega, \Delta)$ if:

a. $F \in T P_{\delta}^{\alpha}(\Omega, \Delta)$,

b. $(\forall x \in \Omega)\left[\left.\widetilde{F}^{(k)}(x)\right|_{,\Delta(x)}=\partial^{k} F(x), \quad k=0,1\right]$.

Thus, periodic approximation $\widetilde{F}^{(k)}$ of $\partial^{k} F$ in $\Delta(x)$ is a constant function for every $x \in \Omega$.

Function $\phi \in \mathrm{H}^{\alpha}(\Omega)$ is the highly oscillating function (with respect to cell $\Delta$ and tolerance parameter $\delta), \phi \in H O_{\delta}^{\alpha}(\Omega, \Delta)$ if:

a. $\phi \in T P_{\delta}^{\alpha}(\Omega, \Delta)$,

b. $(\forall x \in \Omega)\left[\left.\widetilde{\phi}^{(k)}(x)\right|_{,\Delta(x)}=\partial^{k} \widetilde{\phi}(x), \quad k=0,1\right]$.

Function $f \equiv \phi F \in T P_{\delta}^{\alpha}(\Omega, \Delta)$, for every $F \in S V_{\delta}^{\alpha}(\Omega, \Delta)$, satisfies the condition:

c. $\left.\widetilde{f}^{(1)}(x)\right|_{,\Delta(x)}=\left.F(x) \partial^{1} \widetilde{\phi}(x)\right|_{\Delta(x)}$, if $\alpha=0$, we denote $\widetilde{f} \equiv \widetilde{f}^{(0)}$.

Let us denote by $h(\cdot)$ and $g(\cdot)$ highly oscillating, continuous functions, $h, g \in H O_{\delta}^{1}(\Omega, \Delta)$, defined on $\bar{\Omega}$, which have piecewise continuous and bounded gradients $\partial^{1} h, \partial^{1} g$. Functions $h(\cdot), g(\cdot)$ are the fluctuation shape functions of the $1^{\text {st }}$ kind, if they satisfy conditions:

a. $\partial^{k} h \in O\left(l^{\alpha-k}\right), \partial^{k} g \in O\left(l^{\alpha-k}\right)$, for $k=0,1, \alpha=1, \partial^{0} h \equiv h, \partial^{0} g \equiv g$,

b. $\langle h>(x) \approx 0,<g>(x) \approx 0$, for every $x \in \Omega$,

and depend on $l$ as a parameter. Set of all fluctuation shape functions of the $1^{\text {st }}$ kind is denoted by $F S_{\delta}^{1}(\Omega, \Delta)$.

\section{TOLERANCE MODELLING}

\subsection{Fundamental assumptions}

The tolerance modelling is based on two basic assumptions, cf. [18, 17, 2]. The first is the decomposition of the micro-macro, where it is assumed that the fundamental unknowns (in the present issue - the displacement field and the temperature field) can be taken as a sum of averaged part (averaged field) and oscillating part. Furthermore it is assumed that the oscillating part can be expressed as a product of a known oscillating function, called the fluctuation shape function, and the new unknown, the fluctuation amplitude. It is assumed that the new basic unknowns, the averaged fields and the amplitudes of fluctuations, are slowlyvarying functions of that co-ordinate which parameterizes the perpendicular direction to the laminas. For the displacement and for the temperature, respectively:

$$
\begin{gathered}
u_{i}(x, \mathbf{x}, t)=w_{i}(x, \mathbf{x}, t)+h(x) v_{i}(x, \mathbf{x}, t) \\
\Theta(x, \mathbf{x}, t)=\theta(x, \mathbf{x}, t)+g(x) \psi(x, \mathbf{x}, t)
\end{gathered}
$$


and $w_{i}(\cdot, \mathbf{x}, t), v_{i}(\cdot, \mathbf{x}, t), \theta(\cdot, \mathbf{x}, t), \psi(\cdot, \mathbf{x}, t) \in S V_{\delta}^{1}(\Omega, \Delta) \quad$ for every $t ; \quad h(\cdot), g(\cdot) \in F S_{\delta}^{1}(\Omega, \Delta)$. Functions $w_{i}(\cdot, \mathbf{x}, t)$ and $\theta(\cdot, \mathbf{x}, t)$, are the basic unknowns, called the macrodisplacements and the macrotemperature, respectively; $v_{i}(\cdot, \mathbf{x}, t)$ and $\psi(\cdot, \mathbf{x}, t)$ are additional basic unknowns, called the fluctuation amplitudes of displacements and the fluctuation amplitudes of temperature, respectively; $h(\cdot)$ and $g(\cdot)$ are the known fluctuation shape functions. The fluctuation shape functions $h(x), g(x), x \in \bar{\Omega}$, can be given in the following form:

$$
h(x)=g(x)= \begin{cases}{\left[-l \sqrt{3} \frac{v(\bar{x})}{v^{\prime}(\bar{x})}\left(2 x / l+v^{\prime \prime}(\bar{x})\right)\right]} & \text { for } x \in\left[-l / 2,-l / 2+l v^{\prime}(\bar{x})\right], \\ {\left[l \sqrt{3} \frac{v(\bar{x})}{v^{\prime \prime}(\bar{x})}\left(2 x / l-v^{\prime \prime}(\bar{x})\right)\right]} & \text { for } x \in\left[-l / 2+l v^{\prime}(\bar{x}), l / 2\right],\end{cases}
$$

where $\bar{x}$ is a centre of $\Delta=(-l / 2, l / 2)$. Because the non-homogeneity ratio $v(\cdot)$ is a slowlyvarying function the mean values of $h$ and $g$ in every lamina are equal to zero. The shape of this function, $g(x)$ takes into account discontinuities of gradient of temperature field on intersections. For the displacement field it is the most common fluctuations shape function, $h(x)$ considered in such problems.

The second assumption is so-called the tolerance averaging approximation in which it is assumed that some components $O(\delta)$ are negligible small, e.g. in formulas:

$$
\begin{aligned}
& <f>(x)=<\bar{f}>(x)+O(\delta), \\
& <f F>(x)=<f>(x) F(x)+O(\delta), \\
& <f \partial(h F)>(x)=<f \partial h>(x) F(x)+O(\delta),
\end{aligned}
$$

where $x \in \Omega, 0<\delta<<1, f \in T P_{\delta}^{1}(\Omega, \Delta), F \in S V_{\delta}^{1}(\Omega, \Delta), h \in F S_{\delta}^{1}(\Omega, \Delta)$.

\subsection{Modelling procedure}

The modelling procedure is outlined in Woźniak [17]. The starting point is the formulation of the extended action functional:

$$
\begin{aligned}
& \mathrm{A}\left(u_{i}(\cdot), \Theta(\cdot), p_{i}(\cdot), f(\cdot)\right)= \\
& \quad=\int_{\Omega} \int_{\Xi} \int_{t_{0}}^{t_{1}} \Lambda\left(z, \partial_{j} u_{i}(z, \xi, t), u_{i}(z, \xi, t), \partial_{i} \Theta(z, \xi, t), \Theta(z, \xi, t), p_{i}(z, \xi, t), f(z, \xi, t) d t d \xi d z,\right.
\end{aligned}
$$

where $\Lambda\left(\cdot \partial_{j} u_{i}, u_{i}, \partial_{i} \Theta, \Theta, p_{i}, f\right) \in H O_{\delta}^{0}(\Omega, \Delta), p_{i}(\cdot, \xi, t), f(\cdot, \xi, t) \in H O_{\delta}^{0}(\Omega, \Delta)$.

Lagrangian $\Lambda$ is given by the formula:

$$
\Lambda=\frac{1}{2}\left(\rho \dot{u_{i}} \dot{u}_{j} \delta_{i j}-c_{i j k l} \partial_{j} u_{i} \partial_{l} u_{k}+k_{i j} \partial_{i} \Theta \partial_{j} \Theta\right)+p_{i} u_{i}+f \Theta .
$$

The constitutive equations determine $p_{i}$ and $f$ :

$$
p_{i}=\partial_{j}\left(b_{i j} \Theta\right), \quad f=c \dot{\Theta}+T_{0} b_{i j} \partial_{j} \dot{u}_{i} .
$$

For Lagrangian (7) we can write the Euler-Lagrange equations:

$$
\frac{\partial}{\partial t} \frac{\partial \Lambda}{\partial \dot{u}_{i}}+\partial_{j} \frac{\partial \Lambda}{\partial \partial_{j} u_{i}}-\frac{\partial \Lambda}{\partial u_{i}}=0, \quad \partial_{j} \frac{\partial \Lambda}{\partial \partial_{j} \Theta}-\frac{\partial \Lambda}{\partial \Theta}=0 .
$$


The fundamental equations of thermoelasticity problems (1) are obtained by using the extended principle of stationary action from equations (9) combined with equations (7) and (8). Then, we apply the tolerance modelling to action functional (6). Substituting micro-macro decompositions (3) and (4) to formula (6) and using averaging operator (2) to this action functional, we arrive at the tolerance averaged functional $\mathrm{A}\left(u_{i}(\cdot), \Theta(\cdot), p_{i}(\cdot), f(\cdot)\right)$ :

$$
\mathrm{A}_{h g}\left(w_{i}, v_{i}, \theta, \psi, p_{i}, f\right)=\int_{\Omega} \int_{\Xi} \int_{t_{0}}^{t_{1}}<\Lambda_{h g}>\left(x, \partial_{j} w_{i}, w_{i}, \partial_{\alpha} v_{i}, v_{i}, \partial_{i} \theta, \theta, \partial_{\alpha} \psi, \psi, p_{i}, f\right) d t d \xi d x,
$$

where the averaged form $\left\langle\Lambda_{h g}>\right.$ of lagrangian (7) can be written as:

$$
\begin{aligned}
& <\Lambda_{h g}>=\frac{1}{2}<k_{i j}>\partial_{i} \theta \partial_{j} \theta+\frac{1}{2}<k_{i 1} \partial g>\partial_{i} \theta \psi+\frac{1}{2}<k_{j 1} \partial g>\partial_{j} \theta \psi+\frac{1}{2}<k_{\alpha \beta} g g>\partial_{\alpha} \psi \partial_{\beta} \psi+ \\
& +\frac{1}{2}<k_{11} \partial g \partial g>\psi \psi+\frac{1}{2}<\rho>\dot{w}_{i} \dot{w}_{j} \delta_{i j}+\frac{1}{2}<\rho h h>\dot{v}_{i} \dot{v}_{j} \delta_{i j}-\frac{1}{2}<c_{i j k l}>\partial_{j} w_{i} \partial_{l} w_{k}-\frac{1}{2}<c_{i j k l} \partial h>\partial_{j} w_{i} v_{k}- \\
& -\frac{1}{2}<c_{i 1 k l} \partial h>v_{i} \partial_{l} w_{k}-\frac{1}{2}<c_{i 1 k l} \partial h \partial h>v_{i} v_{k}-\frac{1}{2}<c_{i \alpha k \beta} h h>\partial_{\alpha} v_{i} \partial_{\beta} v_{k}+ \\
& +<p_{i}>w_{i}+\leq p_{i} h>v_{i}+<f>\theta+<f g>\psi,
\end{aligned}
$$

and $\left\langle p_{i}>,\left\langle p_{i} h>,\langle f>,\langle f g>\right.\right.$ are defined by the constitutive equations:

$$
\begin{aligned}
& <f>=<c>\dot{\theta}+<T_{0} b_{i j}>\partial_{j} \dot{w}_{i}+<T_{0} b_{i 1} \partial h>\dot{v}_{i}, \\
& <f g>=<c g>\dot{\psi}+<T_{0} b_{i \beta} h g>\partial_{\beta} \dot{v}_{i}, \\
& <p_{i}>=\partial b_{i j} \theta+<b_{i j}>\partial_{j} \theta, \\
& <p_{i} h>=-<b_{i 1} \partial g>\theta+<b_{i \beta} g h>\partial_{\beta} \psi .
\end{aligned}
$$

Underlined terms in formulas (11) and (12) depend on the microstructure parameter $l$. Using the extended principle of stationary action to $\Lambda_{h g}$ the following system of equations with slowly-varying coefficient is obtained:

$$
\begin{aligned}
& \frac{\partial}{\partial t} \frac{\partial<\Lambda_{h g}>}{\partial \dot{w}_{i}}+\partial_{j} \frac{\partial<\Lambda_{h g}>}{\partial \partial w_{i}}-\frac{\partial<\Lambda_{h g}>}{\partial w_{i}}=0, \\
& \frac{\partial}{\partial t} \frac{\partial<\Lambda_{h g}>}{\partial \dot{v}_{i}}+\partial_{\alpha} \frac{\partial<\Lambda_{h g}>}{\partial \partial_{\alpha} v_{i}}-\frac{\partial<\Lambda_{h g}>}{\partial v_{i}}=0, \\
& \partial_{j} \frac{\partial<\Lambda_{h g}>}{\partial \partial_{j} \theta}-\frac{\partial<\Lambda_{h g}>}{\partial \theta}=0, \quad \partial_{\alpha} \frac{\partial<\Lambda_{h g}>}{\partial \partial_{\alpha} \psi}-\frac{\partial<\Lambda_{h g}>}{\partial \psi}=0,
\end{aligned}
$$

\section{GOVERNING EQUATIONS}

\subsection{The tolerance model equations}

The following form of averaged equations for thermoelasticity problems is obtained by combining equations (13) with formulas (11) and (12):

$$
\begin{aligned}
& \partial_{j}\left(<c_{i j k l}>\partial_{l} w_{k}+<c_{i j k 1} \partial h>v_{k}\right)-<\rho>\ddot{w}_{i}=\partial<b_{i j}>\theta+<b_{i j}>\partial_{j} \theta, \\
& <c_{i 1 k l} \partial h>\partial_{l} w_{k}-\partial_{\alpha}\left(<c_{i \alpha k \beta} h h>\partial_{\beta} v_{k}\right)+<c_{i 1 k 1} \partial h \partial h>v_{k}+<\rho h h>\ddot{v}_{i}=<b_{i 1} \partial h>\theta-<b_{i \beta} g h>\partial_{\beta} \psi, \\
& \partial_{j}\left(<k_{i j}>\partial_{i} \theta+<k_{1 j} \partial g>\psi\right)=<c>\dot{\theta}+<T_{0} b_{i j}>\partial_{j} \dot{w}_{i}+<T_{0} b_{i 1} \partial h>\dot{v}_{i}, \\
& \underline{\partial_{\alpha}\left(<k_{\alpha \beta} g g>\partial_{\beta} \psi\right)-<k_{i 1} \partial g>\partial_{i} \theta-<k_{11} \partial g \partial g>\psi=\underline{\operatorname{cog}>\psi}+\underline{T_{0}} b_{i \beta} h g>\partial_{\beta} \dot{v}_{i},}
\end{aligned}
$$

where the underlined terms depend on the microstructure parameter $l$. 
In the above equations (14) the coefficients are slowly-varying functions in $x$, in contrast to equations (1), where the coefficients are non-continuous, highly oscillating and toleranceperiodic functions. Equations (14) together with micro-macro decompositions (3) and (4) constitute the tolerance model of thermoelasticity problems for transversally graded laminates. It is necessary to formulate boundary conditions for the macrodisplacements $w_{i}$ and the macrotemperature $\theta$ on the edges $x=0, H$ and $x_{\alpha}=0, L_{\alpha}$, but for the fluctuation amplitudes of displacements $v_{i}$ and the fluctuation amplitude of temperature $\psi-$ only on the edges $x_{\alpha}=0, L_{\alpha}$.

\subsection{The asymptotic model equations}

Equations of the asymptotic model can be obtained directly from equations of the tolerance model, neglecting underlined terms (involving the microstructure parameter $l$ ):

$$
\begin{aligned}
& \partial_{j}\left(<c_{i j k l}>\partial_{l} w_{k}+<c_{i j k 1} \partial h>v_{k}\right)-<\rho>\ddot{w}_{i}=\partial<b_{i j}>\theta+<b_{i j}>\partial_{j} \theta, \\
& <c_{i k k l} \partial h>\partial_{l} w_{k}+<c_{i l k k} \partial h \partial h>v_{k}=<b_{i 1} \partial h>\theta, \\
& \partial_{j}\left(<k_{i j}>\partial_{i} \theta+<k_{1 j} \partial g>\psi\right)=<c>\theta+<T_{0} b_{i j}>\partial_{j} \dot{w}_{i}+<T_{0} b_{i 1} \partial h>\dot{v}_{i}, \\
& <k_{i 1} \partial g>\partial_{i} \theta+<k_{11} \partial g \partial g>\psi=0,
\end{aligned}
$$

The above equations describe the thermoelastic effects in laminates on the macro-level.

\section{EXAMPLE}

The considered laminate with transversally graded material properties has a height equals $H=0.5 \mathrm{~m}$, thickness of the lamina $l=0.01 \mathrm{~m}$. It is assumed that the both materials are isotropic and homogeneous. Hence, let us denote by indexes 1, 2 properties of the first and the second material: Young's moduli $E_{1}, E_{2}$, Poisson's ratios $v_{1}, v_{2}$, mass densities $\rho_{1}, \rho_{2}$, expansion coefficients $\alpha_{1}, \alpha_{2}$, specific heats $c_{1}, c_{2}$, heat conductions $k_{1}, k_{2}$, Lame's constants $\lambda_{1}, \lambda_{2}, \mu_{1}$, $\mu_{2}$, thermoelasticity constants $b_{1}, b_{2}$. So for the first material (steel) there are: $E_{1}=210 \cdot 10^{9}[\mathrm{~Pa}]$, $v_{1}=0.3[-], \quad \rho_{1}=7800\left[\mathrm{~kg} / \mathrm{m}^{3}\right], \quad \alpha_{1}=10.8 \cdot 10^{-6}[1 / \mathrm{K}], \quad c_{1}=460[\mathrm{~J} /(\mathrm{kgK})], \quad k_{1}=58\left[\mathrm{~W} /\left(\mathrm{m}^{2} \mathrm{~K}\right)\right]$, $\lambda_{1}=1.21 \cdot 10^{11}[\mathrm{~Pa}], \quad \mu_{1}=8.08 \cdot 10^{10}[\mathrm{~Pa}], \quad b_{1}=5.67 \cdot 10^{6}[\mathrm{~Pa} / \mathrm{K}]$ and for the second material (aluminum): $\quad E_{2}=69 \cdot 10^{9}[\mathrm{~Pa}], \quad v_{2}=0.33[-], \quad \rho_{2}=2720\left[\mathrm{~kg} / \mathrm{m}^{3}\right], \quad \alpha_{2}=23.1 \cdot 10^{\cdot 6}[1 / \mathrm{K}]$, $c_{2}=920[\mathrm{~J} /(\mathrm{kgK})], \quad k_{2}=229\left[\mathrm{~W} /\left(\mathrm{m}^{2} \mathrm{~K}\right)\right], \quad \lambda_{2}=5.0354 \cdot 10^{10}[\mathrm{~Pa}], \quad \mu_{2}=2.5940 \cdot 10^{10}[\mathrm{~Pa}]$, $b_{2}=4.6879 \cdot 10^{6}[\mathrm{~Pa} / \mathrm{K}]$. The fluctuation shape functions $h(x), g(x)$ are given by formulas $(5)$.

The six different distribution functions of material properties are considered:

$$
\begin{aligned}
& v_{n 0}^{\prime}=0.5, v_{n 1}^{\prime}=x_{1} / H, v_{n 2}^{\prime}=\left(x_{1}\right)^{2} / H^{2}, v_{n 3}^{\prime}=\left(x_{1}\right)^{3} / H^{3}, \\
& v_{n 4}^{\prime}=\left(1-e^{\frac{2 x_{1}}{H}}\right) /\left(1-e^{2}\right), v_{n 5}^{\prime}=\frac{1}{2} \ln \left[\left(e^{2}-1\right) x_{1} / H+1\right] .
\end{aligned}
$$

The following assumptions are taken into account: stationary, one-dimensional issue, loads are a temperature: $\left.\theta_{0}\right|_{x_{1}=0}=90^{\circ} \mathrm{C},\left.\theta_{0}\right|_{x_{1}=H}=10^{\circ} \mathrm{C}$, and two types of boundary conditions: $1^{\circ}:\left.w_{1}^{1}\right|_{x_{1}=0}=0,\left.\sigma_{1}\right|_{x_{1}=H}=0,2^{\circ}:\left.w_{1}^{2}\right|_{x_{1}=0}=0,\left.w_{1}^{2}\right|_{x_{1}=H}=0$.

Using the tolerance model equations (14) we obtain the equations for considered issue: 


$$
\begin{array}{ll}
\partial\left(<c_{1111}>\partial w_{1}+<c_{1111} \partial h>v_{1}\right)=\partial<b_{11}>\theta+<b_{11}>\partial \theta, & \text { (a) } \\
<c_{111} \partial h \partial h>v_{1}+<c_{111} \partial h>\partial w_{1}=<b_{11} \partial h>\theta, & \text { (b) } \\
\partial\left(<k_{11}>\partial \theta+<k_{11} \partial g>\psi\right)=0, & \text { (c) } \\
<k_{11} \partial g>\partial \theta+<k_{11} \partial g \partial g>\psi=0 . & \text { (d) }
\end{array}
$$

Using equations (16d) and (16b) we obtained the formulas for the fluctuation amplitudes of temperature and the fluctuation amplitudes of displacements, respectively:

$$
\begin{gathered}
\psi=-\frac{<k_{11} \partial g>\partial_{1} \theta}{<k_{11} \partial g \partial g>}, \\
v_{1}=\frac{<b_{11} \partial h>\theta-<c_{1111} \partial h>\partial_{1} w_{1}}{<c_{1111} \partial h \partial h>} .
\end{gathered}
$$

Then, solving (in analytical way) equations (16c) and (16a), we obtain the formulas for the macrotemperature (after substituting the load conditions) and the macrodisplacements for the $1^{\circ}$ and the $2^{\circ}$ boundary conditions, respectively:

$$
\begin{gathered}
\theta\left(x_{1}\right)=\theta_{0}+\left(\theta_{H}-\theta_{0}\right) \frac{k_{1} x_{1}+\left(k_{2}-k_{1}\right) \int_{0}^{x_{1}} v^{\prime}(s) d s}{k_{1} H+\left(k_{2}-k_{1}\right) \int_{0}^{H} v^{\prime}(s) d s}, \\
w_{1}^{1}\left(x_{1}\right)=w_{0}+\left(w_{H}-w_{0}\right) \frac{\int_{0}^{x_{1}}<c^{-1}>(s) d s}{\int_{0}^{H}<c^{-1}>(s) d s}+ \\
+\int_{0}^{H} \theta(s)<b c^{-1}>(s) d s\left(\frac{\int_{0}^{x_{1}} \theta(s)<b c^{-1}>(s) d s}{\int_{0}^{H} \theta(s)<b c^{-1}>(s) d s}-\frac{\int_{0}^{x_{1}}<c^{-1}>(s) d s}{\left.\int_{0}^{H}<c^{-1}>(s) d s\right)},\right. \\
w_{1}^{2}\left(x_{1}\right)=w_{0}+\theta_{H}<b c^{-1}>(H) \int_{0}^{x_{1}}\left[\frac{\theta(s)<b c^{-1}>(s)}{\theta(H)<b c^{-1}>(H)}-\frac{<c^{-1}>(s)}{<c^{-1}>(H)}\right] d s+\sigma_{H} \int_{0}^{x_{1}} \frac{<c^{-1}>(s)}{<c^{-1}>(H)} d s .
\end{gathered}
$$

After taking into account the specific distribution functions of materials, we obtain the formulas and graphs for the displacement and the temperature.

For the distribution function of material $v_{n 0}^{\prime}=0.5$ :

$$
\begin{gathered}
\theta\left(x_{1}\right)=\theta_{0}+\frac{\theta_{H}-\theta_{0}}{H} x_{1}, \\
w_{1}^{1}\left(x_{1}\right)=x_{1}\left[\frac{<b_{11}><c_{1111} \partial h \partial h>-<b_{11} \partial h><c_{1111} \partial h>}{2\left(<c_{1111}><c_{1111} \partial h \partial h>-<c_{1111} \partial h>^{2}\right)}\left(\theta_{H}-\theta_{0}\right) \frac{x_{1}}{H}+\theta_{0}\right], \\
w_{1}^{2}\left(x_{1}\right)=\frac{<b_{11}><c_{1111} \partial h \partial h>-<b_{11} \partial h><c_{1111} \partial h>}{2\left(<c_{1111}><c_{1111} \partial h \partial h>-<c_{1111} \partial h>^{2}\right)}\left(\theta_{H}-\theta_{0}\right) x_{1}\left(\frac{x_{1}}{H}-1\right) .
\end{gathered}
$$

For the distribution function of material $v_{n 1}^{\prime}=x_{1} / H$ :

$$
\theta\left(x_{1}\right)=\theta_{0}+\left(\theta_{H}-\theta_{0}\right) \frac{k_{1} x_{1}+\frac{1}{2}\left(k_{2}-k_{1}\right)\left(x_{1}\right)^{2} H^{-1}}{k_{1} H+\frac{1}{2}\left(k_{2}-k_{1}\right) H},
$$




$$
\begin{aligned}
& w_{1}^{i}\left(x_{1}\right)=\frac{\mathrm{A}}{c_{2}} x_{1}+\mathrm{A} \frac{c_{2}-c_{1}}{c_{1} c_{2}} \frac{1}{2} \frac{\left(x_{1}\right)^{2}}{H}+\frac{b_{2}}{c_{2}}\left[\theta_{0} x_{1}+\frac{\theta_{H}-\theta_{0}}{k_{1} H+\left(k_{2}-k_{1}\right) \frac{1}{2} H}\left(\frac{1}{2} k_{1}\left(x_{1}\right)^{2}+\frac{1}{6} \frac{\left(k_{2}-k_{1}\right)\left(x_{1}\right)^{3}}{H}\right)\right]+ \\
& +\frac{b_{1} c_{2}-b_{2} c_{1}}{c_{1} c_{2}}\left(\frac{1}{4} \frac{\mathrm{a}_{1}}{H}\left(x_{1}\right)^{4}+\frac{1}{3} \frac{\mathrm{a}_{2}}{H}\left(x_{1}\right)^{3}+\frac{1}{2} \frac{\mathrm{a}_{3}}{H}\left(x_{1}\right)^{2}\right)+\mathrm{B}
\end{aligned}
$$

where: A, B are constants, which depend on the boundary conditions; $\mathrm{a}_{1}=\frac{\theta_{H}-\theta_{0}}{H^{2}} \frac{k_{2}-k_{1}}{k_{1}+k_{2}}, \quad \mathrm{a}_{2}=\frac{2\left(\theta_{H}-\theta_{0}\right) k_{1}}{H\left(k_{1}+k_{2}\right)}, \quad \mathrm{a}_{3}=\theta_{0}$.

For the distribution function of material $v_{n 2}^{\prime}=\left(x_{1}\right)^{2} H^{-2}$ :

$$
\begin{gathered}
\theta\left(x_{1}\right)=\theta_{0}+\left(\theta_{H}-\theta_{0}\right) \frac{k_{1} x_{1}+\frac{1}{3}\left(k_{2}-k_{1}\right)\left(x_{1}\right)^{3} H^{-2}}{k_{1} H+\frac{1}{3}\left(k_{2}-k_{1}\right) H}, \\
w_{1}^{i}\left(x_{1}\right)=\frac{\mathrm{A}}{c_{2}} x_{1}+\mathrm{A} \frac{c_{2}-c_{1}}{c_{1} c_{2}} \frac{1}{3} \frac{\left(x_{1}\right)^{3}}{H^{2}}+\frac{b_{2}}{c_{2}}\left[\theta_{0} x_{1}+\frac{\theta_{H}-\theta_{0}}{k_{1} H+\left(k_{2}-k_{1}\right) \frac{1}{3} H}\left(\frac{1}{2} k_{1}\left(x_{1}\right)^{2}+\frac{1}{12} \frac{\left(k_{2}-k_{1}\right)\left(x_{1}\right)^{4}}{H^{2}}\right)\right]+ \\
+\frac{b_{1} c_{2}-b_{2} c_{1}}{c_{1} c_{2}}\left(\frac{1}{6} \frac{\mathrm{a}_{1}}{H^{2}}\left(x_{1}\right)^{6}+\frac{1}{5} \frac{\mathrm{a}_{2}}{H^{2}}\left(x_{1}\right)^{5}+\frac{1}{4} \frac{\mathrm{a}_{3}}{H^{2}}\left(x_{1}\right)^{4}+\frac{1}{3} \frac{\mathrm{a}_{4}}{H^{2}}\left(x_{1}\right)^{3}\right)+\mathrm{B},
\end{gathered}
$$

where: A, B are constants, which depend on the boundary conditions; $\mathrm{a}_{1}=\frac{\theta_{H}-\theta_{0}}{H^{3}} \frac{k_{2}-k_{1}}{2 k_{1}+k_{2}}, \quad \mathrm{a}_{2}=0, \quad \mathrm{a}_{3}=\frac{3\left(\theta_{H}-\theta_{0}\right) k_{1}}{H\left(2 k_{1}+k_{2}\right)}, \quad \mathrm{a}_{4}=\theta_{0}$.
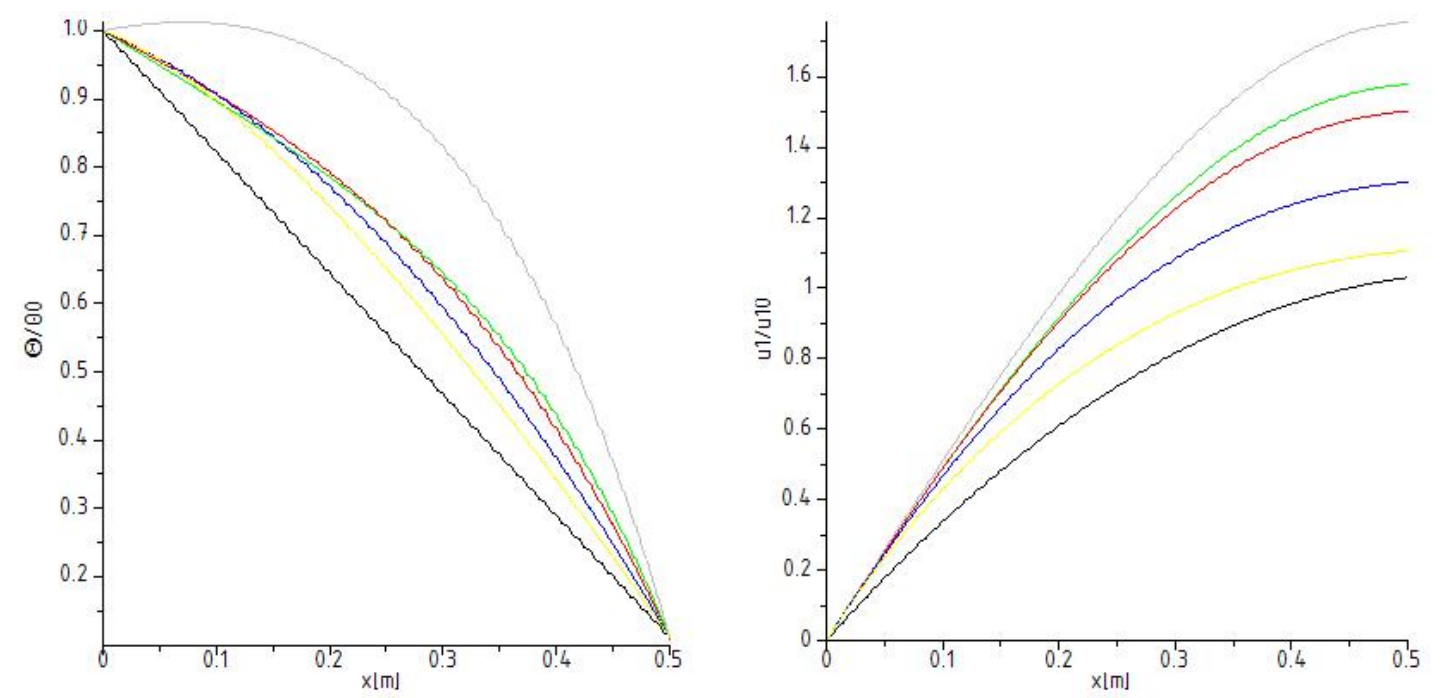

Figure 2: a. The temperature; $b$. The displacements - for the boundary conditions $1^{\circ}$ and various distribution functions of material properties: $v_{\mathrm{n} 0}^{\prime}, v_{\mathrm{n} 1}^{\prime}, v_{\mathrm{n} 2}^{\prime}, v_{\mathrm{n} 3}^{\prime}, v_{\mathrm{n} 4}^{\prime}$,

For the distribution function of material $v_{n 3}^{\prime}=\left(x_{1}\right)^{3} H^{-3}$ :

$$
\theta\left(x_{1}\right)=\theta_{0}+\left(\theta_{H}-\theta_{0}\right) \frac{k_{1} x_{1}+\frac{1}{4}\left(k_{2}-k_{1}\right)\left(x_{1}\right)^{4} H^{-3}}{k_{1} H+\frac{1}{4}\left(k_{2}-k_{1}\right) H},
$$




$$
\begin{aligned}
& w_{1}^{i}\left(x_{1}\right)=\frac{\mathrm{A}}{c_{2}} x_{1}+\mathrm{A} \frac{c_{2}-c_{1}}{c_{1} c_{2}} \frac{1}{4} \frac{\left(x_{1}\right)^{4}}{H^{3}}+\frac{b_{2}}{c_{2}}\left[\theta_{0} x_{1}+\frac{\theta_{H}-\theta_{0}}{k_{1} H+\left(k_{2}-k_{1}\right) \frac{1}{4} H}\left(\frac{1}{2} k_{1}\left(x_{1}\right)^{2}+\frac{1}{20} \frac{\left(k_{2}-k_{1}\right)\left(x_{1}\right)^{5}}{H^{3}}\right)\right]+ \\
& +\frac{b_{1} c_{2}-b_{2} c_{1}}{c_{1} c_{2}}\left(\frac{1}{8} \frac{\mathrm{a}_{1}}{H^{3}}\left(x_{1}\right)^{8}+\frac{1}{7} \frac{\mathrm{a}_{2}}{H^{3}}\left(x_{1}\right)^{7}+\frac{1}{6} \frac{\mathrm{a}_{3}}{H^{3}}\left(x_{1}\right)^{6}+\frac{1}{5} \frac{\mathrm{a}_{4}}{H^{3}}\left(x_{1}\right)^{5}+\frac{1}{4} \frac{\mathrm{a}_{5}}{H^{3}}\left(x_{1}\right)^{4}\right)+\mathrm{B},
\end{aligned}
$$

where: A, B are constants, which depend on the boundary conditions; $\mathrm{a}_{1}=\frac{\theta_{H}-\theta_{0}}{H^{4}} \frac{k_{2}-k_{1}}{3 k_{1}+k_{2}}, \quad \mathrm{a}_{2}=0, \quad \mathrm{a}_{3}=0, \quad \mathrm{a}_{4}=\frac{4\left(\theta_{H}-\theta_{0}\right) k_{1}}{H\left(3 k_{1}+k_{2}\right)}, \quad \mathrm{a}_{5}=\theta_{0}$.
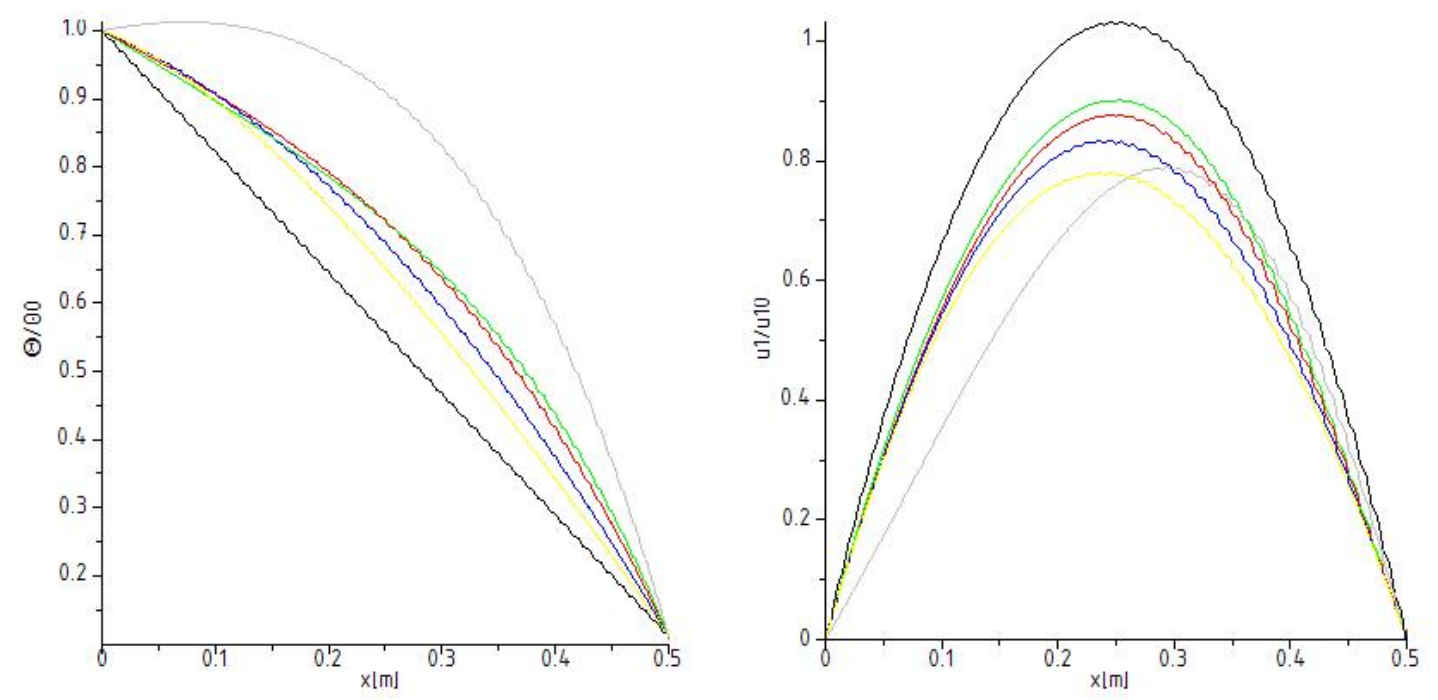

Figure 3: a. The temperature; $b$. The displacements - for the boundary conditions $2^{\circ}$ and various distribution functions of material properties $v_{\mathrm{n} 0}^{\prime}, v_{\mathrm{n} 1}^{\prime}, v_{\mathrm{n} 2}^{\prime}, v_{\mathrm{n} 3}^{\prime}, v_{\mathrm{n} 4}^{\prime}$,

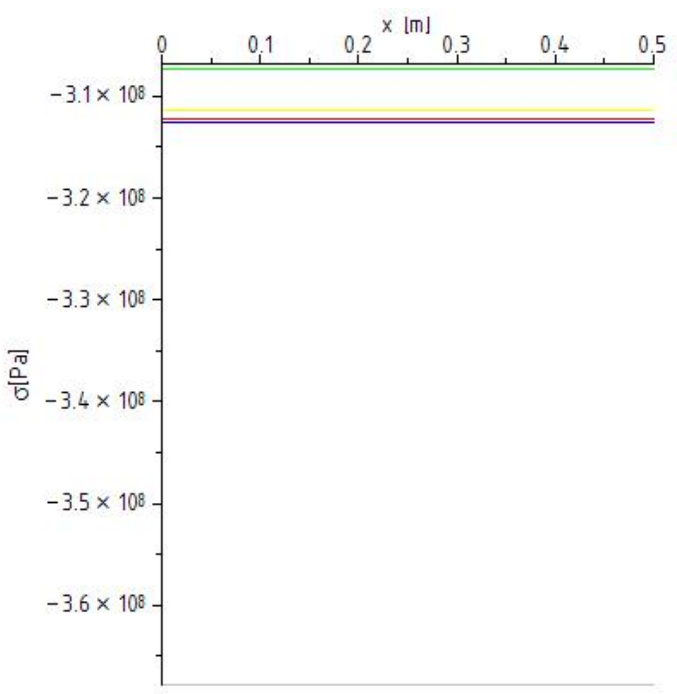

Figure 4: The stresses for the boundary conditions $2^{\circ}$

For the distribution function of material $v_{n 4}^{\prime}=\left(1-\mathrm{e}^{2 x_{1} / H}\right) /\left(1-\mathrm{e}^{2}\right)$ : 


$$
\theta\left(x_{1}\right)=\theta_{0}+\left(\theta_{H}-\theta_{0}\right) \frac{k_{1} x_{1}+\left(k_{2}-k_{1}\right)\left(x_{1}-\frac{1}{2} H e^{\frac{2 x_{1}}{H}}+\frac{1}{2} H\right)}{k_{1} H+\left(k_{2}-k_{1}\right)\left(\frac{3}{2} H-\frac{1}{2} H e^{2}\right)} .
$$

For the distribution function of material $v_{n 5}^{\prime}=\frac{1}{2} \ln \left[\left(e^{2}-1\right) x_{1} H^{-1}+1\right]$ :

$$
\theta\left(x_{1}\right)=\theta_{0}+\left(\theta_{H}-\theta_{0}\right) \frac{k_{1} x_{1}+\left(k_{2}-k_{1}\right) \mathrm{C}}{k_{1} H+\left(k_{2}-k_{1}\right) \mathrm{D}},
$$

where:

$$
\begin{aligned}
& \mathrm{C}=\frac{1}{2} \frac{H+\left(e^{2}-1\right) x_{1}}{e^{2}-1} \ln \left(H+\left(e^{2}-1\right) x_{1}\right)-\frac{1}{2} x_{1}(1+\ln H)-\frac{1}{2} \frac{H}{e^{2}-1} \ln H, \\
& \mathrm{D}=\frac{1}{2} \frac{H+\left(e^{2}-1\right) H}{e^{2}-1} \ln \left(H+\left(e^{2}-1\right) \mathrm{H}\right)-\frac{1}{2} H(1+\ln H)-\frac{1}{2} \frac{H}{e^{2}-1} \ln H .
\end{aligned}
$$

Then, the formula for the stresses has the form:

$$
\sigma_{11}=<c_{1111}>\partial w_{1}+<c_{1111} \partial h>v_{1}-<b_{11}>\theta .
$$

The stresses for the $1^{\circ}$ boundary conditions are equal zero.

\section{REMARKS}

Under the above considerations some general remarks can be formulated:

- using the tolerance modelling it is possible to replace the differential equations of thermo-elasticity for transversally graded laminates with highly-oscillating, noncontinuous coefficients by the differential equations with smooth, slowly-varying coefficients;

- the tolerance model equations take into account the effect of the microstructure size;

- this effect is omitted in the asymptotic model equations;

- the equations for both the models, tolerance and asymptotic, can be applied in the analysis of specific cases, namely where distribution of the ingredients is functional but non-periodic;

- distributions of the temperature and the displacements depend on the distribution functions of material properties.

\section{REFERENCES}

[1] Suresh, S. and Mortensen, A. Fundamentals of functionally graded materials. The University Press, Cambridge, (1998).

[2] Jędrysiak, J. On the tolerance modelling of thermoelasticity problems for transversally graded laminates. Arch. Civ. Mech. Eng. (2011) 11:61-74.

[3] Reiter, T., Dvorak, G.J. and Tvergaard, V. Micromechanical models for graded compositematerials. J. Mech. Phys. Solids (1997) 45:1281-1302.

[4] Jikov, V.V., Kozlov, C.M. and Oleinik, O.A. Homogenization of differential operators and integral functionals. Springer Verlag, Berlin-Heidelberg, (1994).

[5] Matysiak, S.J. On certain problems of heat conduction in periodic composites. Z. Angew. Math. Mech. (1994) 71:524-528.

[6] Kim, K.S. and Noda, N. A Green's function approach to the deflection of a FGM plate 
under transient thermal loading. Arch. Appl. Mech. (2002) 72:127-137.

[7] Ootao, Y. and Tanigawa, Y. Transient thermoelastic analysis for a functionally graded hollow cylinder. J. Therm. Stresses (2006) 29:1031-1046.

[8] Aboudi, J., Pindera, M. J. and Arnolds, M. Higher-order theory for functionally graded materials. Composites (1999) 30:777-832.

[9] Bansal, Y. and Pindera, M. J. Efficients reformulation of the thermoelastic higher-order theory for functionally graded materials. J. Therm. Stresses (2003) 26:1055-1092.

[10] Chiba, R. Stochastic thermal stresses in an FGM annular disc of variable thickness with spatially random heat transfer coefficients. Meccanica (2009) 44:159-176.

[11] Schmauder, S. and Weber, U. Modelling of functionally graded materials by numerical homogenization. Arch. Appl. Mech. (2001) 71:182-192.

[12] Goldberg, R.K. and Hopkins, D.A. Thermal analysis of a functionally graded material subject to a thermal gradient using the boundary element method. Comp. Eng. (1995) 5:793-806.

[13] Sladek, J., Sladek,. V. and Zhang, Ch. Transient heat conduction analysis in functionally graded materials by the meshless local boundary integral equation method. Comput. Mater. Sci. (2003) 28:494-504.

[14] Sadowski, T., Ataya, S. and Nakonieczny, K. Thermal analysis of layered FGM cylindrical plates subjected to sudden cooling process at one side. Comparison of two applied methods for problem solution. Comput. Mater. Sci. (2009) 45:624-632.

[15] Woźniak, C. and Wierzbicki, E. Averaging techniques in thermomechanics of composite solids, Publishing House of Częstochowa Univ. Techn., Częstochowa, (2000).

[16] Jędrysiak, J. Thermomechanics of laminates, plates and shields with functional graded properties (in Polish), Publishing House of Łódź Univ. Techn., Łódź, (2010).

[17] Woźniak, C., et al, (eds.) Mathematical modelling and analysis in continuum mechanics of microstructured media, Publishing House of Silesian Univ. Techn., Gliwice, (2010).

[18] Woźniak, C., Michalak, B. and Jędrysiak, J. (eds.) Thermomechanics of microheterogeneous solids and structures. Tolerance averaging approach, Publishing House of Łódź Univ. Techn., Łódź, (2008).

[19] Ostrowski,. P. and Michalak, B. Non-stationary heat transfer in hollow cylinder with functionally graded material properties. J. Theor. Appl. Mech. (2011) 49.2:385-397.

[20] Ostrowski, P. Thermoelasticity in a two-phase hollow cylinder with longitudinally graded material properties, Shell Structures. Theory and Applications (2014) 3:133-136.

[21]Jędrysiak, J. and Radzikowska, A. On the modelling of heat conduction in a nonperiodically laminated layer. J. Theor. Appl. Mech. (2007) 45:239-257.

[22] Baczyński, Z.F. Dynamic thermoelastic processes in microperiodic composites. J. Therm. Stresses (2003) 26:55-66.

[23] Ignaczak, J. A spatial decay estimate for transient thermoelastic process in a composite semispace. J. Therm. Stresses (2000) 23:1-14. 